\title{
Numerical Study of Entropy Generation in Mixed MHD Convection in a Square Lid-Driven Cavity Filled with Darcy-Brinkman-Forchheimer Porous Medium
}

\author{
Rahma Bouabda ${ }^{1, *}$, Mounir Bouabid ${ }^{2}$, Ammar Ben Brahim ${ }^{1}$ and Mourad Magherbi ${ }^{2}$ \\ 1 Chemical and Process Engineering Department, Engineers National School of Gabès, \\ Applied Thermodynamics Unit, Gabès University, Omar Ibn El Khattab Street, 6029 Gabès, Tunisia; \\ ammar.benbrahim@enig.rnu.tn \\ 2 Chemical Engineering Department, High Institute of Applied Sciences and Technology, Gabès University, \\ Omar Ibn El Khattab Street, 6029 Gabès, Tunisia; bouabid.mpcshun@yahoo.fr (M.B.); \\ magherbim@yahoo.fr (M.M.) \\ * Correspondence: bouabdar@yahoo.com; Tel.: +216-23-293-425 \\ Academic Editor: Brian Agnew \\ Received: 3 October 2016; Accepted: 22 November 2016; Published: 6 December 2016
}

\begin{abstract}
This investigation deals with the numerical simulation of entropy generation at mixed convection flow in a lid-driven saturated porous cavity submitted to a magnetic field. The magnetic field is applied in the direction that is normal to the cavity cross section. The governing equations, written in the Darcy-Brinkman-Forchheimer formulation, are solved using a numerical code based on the Control Volume Finite Element Method. The flow structure and heat transfer are presented in the form of streamlines, isotherms and average Nusselt number. The entropy generation was studied for various values of Darcy number $\left(10^{-3} \leq \mathrm{Da} \leq 1\right)$ and for a range of Hartmann number $\left(0 \leq \mathrm{Ha} \leq 10^{2}\right)$. It was found that entropy generation is affected by the variations of the considered dimensionless physical parameters. Moreover, the form drag related to the Forchheimer effect remains significant until a critical Hartmann number value.
\end{abstract}

Keywords: mixed convection; cavity; porous media; entropy generation; numerical method

\section{Introduction}

The mixed magneto-convection in a lid-driven porous cavity has received extensive attention due to itswide variety of uses in engineering applications, such as the cooling of electronic devices, solar collectors, energy storage, crystal growth, food processing and nuclear reactors. Some text booksthat presented a numerical study on convective flow in porous media areby Nield and Bejan [1], Ingham and Pop [2], Vafai [3], and Bejan et al. [4]. Chamkha [5] presented a numerical work on hydromagnetic convection flow in a lid-driven cavity in the presence of a magnetic field. He found that the flow behavior and the heat transfer characteristics inside the cavity are strongly affected by the presence of the magnetic field.Khanafer and Vafai [6] numerically studied the double mixed convection in a saturated porous lid-driven enclosure filled with a non-Darcian fluid. The influence of the Lewis, Reynolds and Darcy numbers on the heat and mass transfer processes was analyzed. Results revealed that the flow characteristics and the heat transfer are strongly dependent on the Richardson number. Cheng [7] numerically investigated mixed convection in a lid-driven square cavity with the aim to characterizing the heat transfer behavior and to validate the Nusselt number correlations reported in the literature. Mixed convection in a lid-driven porous cavity in the presence of a magnetic field was studied numerically by Muthtamilselvan et al. [8]. It was found that the heat transfer strongly depends 
on the magnetic field strength and the Darcy number. Rahman et al. [9] numerically investigated the Magneto-hydrodynamics mixed convection in a lid-driven square cavity. They showed that the Reynolds number has a significant effect on the streamlines and isotherms for different convective regimes. Mansour et al. [10] analyzed the numerical simulation of mixed convection flow in a square lid-driven cavity partially heated from below using $\mathrm{Cu}$-water, $\mathrm{Ag}$-water, $\mathrm{Al}_{2} \mathrm{O}_{3}$-water and $\mathrm{TiO}_{2}$-water nanofluid. They showed that the increase in the solid volume fraction leads to a decrease in the activity of the fluid motion and the fluid temperature. Hydrodynamic mixed convection in a lid-driven cavity heated from the top with a wavy bottom surface was numerically studied by Saha et al. [11], using the Galerkin finite element method. They observed that the variation in the Reynolds number affects the flow and thermal current activities. Mamourian et al. [12] presented the optimum conditions of the mixed convection heat transfer in a wavy surface square cavity filled with $\mathrm{Cu}$-water nanofluid by utilizing the Taguchi method. They observed that the entropy generation and the mean Nusselt number decrease with the increase of the wavelength of the wavy surface for a given Richardson number. Akbar et al. [13] studied the effect of the entropy and the magnetic field in an endoscope filled with $\mathrm{Cu}$-water. They found that the increase of the Brinkman and the Hartmann numbers tends to increase the temperature. Also, the increase of the Brinkman number induced the increase of the entropy generation. Ellahi et al. [14] investigated the shape effect of the nanoparticles suspended in HFE-7100 over a wedge in the mixed convection. They showed that the entropy generation approaches zero near to the free stream region. Akbar et al. [15] presented the effect of the entropy and the magnetic field on the peristaltic flow of the copper water fluid in an asymmetric horizontal channel. They showed that the entropy generation number increases with the Brinkman number. In the middle of the channel, the flow velocity decreases when the magnetic Reynolds number increases.

The main objective of the present investigation is to examine the effect of a magnetic field on heat transfer and entropy generation in a lid-driven saturated porous cavity, under the Brinkman-Forchheimer extended Darcy formulation. The governing equations of the considered problem are solved using the Control Volume Finite Elements method. The influence of the Hartman, Rayleigh, Reynolds numbers and the Forchheimer parameter, on thermodynamic irreversibility, was studied.

\section{Mathematical Formulation}

Consider a steady-state two-dimensional laminar flow in a lid-driven saturated porous cavity filled with a Newtonian, incompressible and electrically conducting fluid. The square cavity with porosity $(\varepsilon)$ has a height $(\mathrm{H})$ as shown in Figure 1. The top wall is moving from left to right at a constant speed $U_{0}$ and is maintained at a constant temperature $\theta_{c}$, whereas the bottom wall is maintained at a constant temperature $\theta_{\mathrm{h}}\left(\theta_{\mathrm{c}}<\theta_{\mathrm{h}}\right)$. All other remaining walls are adiabatic and insulated. A uniform magnetic field of strength $\left(B_{0}\right)$ is applied in the direction normal to the cavity cross section. The physical properties of the fluid are considered to be constant, except the density variation which is applied in the Boussinesq approximation for the buoyancy term $\left(\rho=\rho_{0}\left[1-\beta_{\theta}\left(\theta-\theta_{0}\right)\right]\right) \cdot \rho_{0}$ is the fluid density at temperature $\theta_{0}$ and $\beta_{\theta}$ is the thermal expansion coefficient.

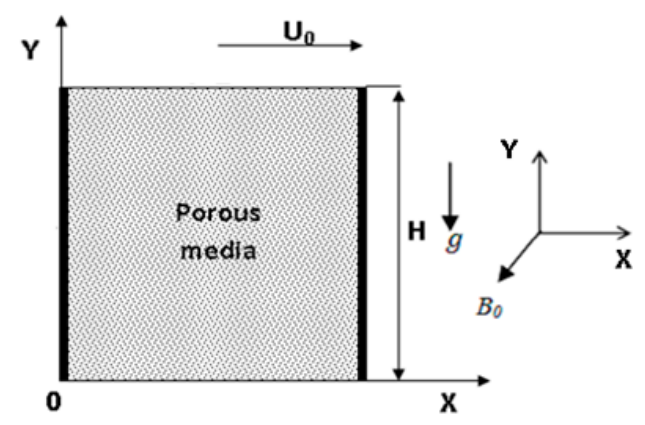

Figure 1. Schematic view of the physical model. 
Using the Darcy-Brinkman-Forchheimer model with the above assumptions, the governing equations of continuity, momentum and energy conservations can be written in dimensionless form as (Nield and Bejan [1]):

Continuity equation:

$$
\frac{\partial U}{\partial X}+\frac{\partial V}{\partial Y}=0
$$

X-Momentum Equation:

$$
\frac{\partial U}{\partial \tau}+d i v J_{U}=-\varepsilon \frac{\partial P}{\partial X}-\frac{\varepsilon}{\operatorname{ReDa}} U-\frac{\varepsilon \mathrm{F}_{\varepsilon}}{\sqrt{\mathrm{Da}}} \sqrt{U^{2}+V^{2}} U-\frac{\varepsilon \mathrm{Ha}^{2}}{\operatorname{Re}} U
$$

Y-Momentum Equation:

$$
\frac{\partial V}{\partial \tau}+d i v J_{V}=-\varepsilon \frac{\partial P}{\partial Y}-\frac{\varepsilon}{\operatorname{ReDa}} V-\frac{\varepsilon \mathrm{F}_{\varepsilon}}{\sqrt{\mathrm{Da}}} \sqrt{U^{2}+V^{2}} V-\frac{\varepsilon \mathrm{Ha}^{2}}{\mathrm{Re}} V-\varepsilon \mathrm{R}_{\mathrm{i}} T
$$

Energy Equation:

$$
\frac{\partial T}{\partial \tau}+\operatorname{div}_{T}=0
$$

With:

$$
\begin{aligned}
J_{U} & =\frac{1}{\varepsilon}(\boldsymbol{U} \cdot \nabla) U-\frac{1}{\operatorname{Re}} \operatorname{grad} U \\
J_{V} & =\frac{1}{\varepsilon}(\boldsymbol{U} \cdot \nabla) V-\frac{1}{\operatorname{Re}} \operatorname{grad} V \\
J_{T} & =\frac{1}{\varepsilon}(\boldsymbol{U} \cdot \nabla) T-\frac{1}{\mathrm{Pe}} \operatorname{grad} T
\end{aligned}
$$

The reference length, velocity, temperature, pressure and time for scaling are the height $\mathrm{H}$ of the cavity, the Lid velocity $U_{0}$, the temperature difference $\left(\theta-\theta_{0}\right)$ between the two horizontal walls of the cavity, $\rho_{0} \mathrm{U}_{0}^{2}$ and $\mathrm{H} / \mathrm{U}_{0}$, respectively. Thus $\mathrm{Ra}=\mathrm{g} \beta_{\theta}\left(\theta_{\mathrm{h}}-\theta_{\mathrm{c}}\right) \mathrm{L}^{3} /(v \alpha)$ is the Rayleigh number, $\mathrm{Re}=\mathrm{U}_{0} \mathrm{H} / \nu$ is the Reynolds number, $\mathrm{Da}=\mathrm{K} / \mathrm{H}^{2}$ is the Darcy number, $\mathrm{Ha}=\mathrm{B}_{0} \mathrm{H} \sqrt{\sigma_{0} / \mu}$ is the Hartman number, $\mathrm{Ri}=\mathrm{Ra} /(\mathrm{PeRe})$ is the Richardson number, $\mathrm{Pe}=\mathrm{RePr}$ is the Peclet number and $\operatorname{Pr}=v / \alpha$ is the Prandtl number. $g$ is the gravity constant.

The initial and boundary conditions, expressed in dimensionless form are:

Initial conditions:

$$
U(X, Y)=V(X, Y)=P=0 ; T(X, Y)=1-Y
$$

Boundary conditions

At the vertical walls

$$
\begin{aligned}
& U(0, Y)=V(0, Y)=0 ; \frac{\partial T}{\partial X}=0 \\
& U(1, Y)=V(1, Y)=0 ; \frac{\partial T}{\partial Y}=0
\end{aligned}
$$

At the horizontal active walls

$$
\begin{aligned}
& U(X, 0)=0 ; V(X, 0)=0 ; T(X, 0)=1 \\
& U(X, 1)=1 ; V(X, 1)=0 ; T(X, 1)=0
\end{aligned}
$$


The heat transfer along the heated wall is given by the average Nusselt number, defined as follows:

$$
\mathrm{Nu}=\int_{0}^{1}-\left(\frac{\partial T}{\partial Y}\right) d X
$$

\section{Second Law Formulation}

The local entropy generation in a porous medium denoted $\left(S_{l}\right)$ can be calculated by following the equation of (Woods [16]):

$$
S_{l}=\left[\left(\frac{\partial T}{\partial X}\right)^{2}+\left(\frac{\partial T}{\partial Y}\right)^{2}\right]+\frac{\mathrm{Br}^{*}}{\mathrm{Da}}\left[U^{2}+V^{2}\right]+\mathrm{Br}^{*}\left[\left(\frac{\partial U}{\partial X}\right)^{2}+\left(\frac{\partial V}{\partial Y}\right)^{2}+\left(\frac{\partial U}{\partial Y}+\frac{\partial V}{\partial X}\right)^{2}\right]+\mathrm{Br}^{*} \mathrm{Ha}^{2}\left[U^{2}+V^{2}\right]
$$

The first term of the right hand side of Equation (8) is the heat transfer irreversibility, the second represents the Darcy viscous irreversibility, the third is related to the clear fluid viscous irreversibility and the fourth is relative to the magnetic effect. The dimensionless total entropy generation $(S)$ is obtained by a numerical integration of the dimensionless local entropy generation over the entire volume of the cavity, $\Omega$. It is given by:

$$
S=\int_{\Omega} S_{l} d \Omega
$$

\section{Numerical Method}

Equations (1)-(4) were solved by the SIMPLE algorithm of Patankar [17] and the SIMPLEC approximation of Van Doormal and Raithby [16]. The used numerical code, described and validated in detail in Abbassi et al. [18], employs the Control Volume Finite Element Method (CVFEM) of Patankar [17] and Saabas and Baliga [19]). Note that the SIMPLE algorithm was developed at Imperial College-London by Pantakar and Spalding [20].

The convergence criterion is given by ( $\chi$ represents $U, V$ or $T$ ):

$$
\max \left|\frac{\chi^{t+\Delta t}-\chi^{t}}{\chi^{t+\Delta t}}\right| \leq 10^{-5}
$$

The local entropy generation is calculated after calculating the temperature and velocity components in each control volume in the mesh. Results shown in the following section have been calculated by taking into account the initial and the boundary conditions (6) given above. In order to validate the accuracy of the proposed computational model, results of the present work are compared to those provided from Muthtamilselvan et al. [8] and Jue [21] for Rayleigh numbers equal to $10^{3}, 10^{4}$ and $10^{5}$. As can be seen in Tables 1 and 2, a good agreement was obtained.

Table 1. Validation of the numerical results.

\begin{tabular}{cccc}
\hline Ra & $\mathbf{1 0}^{\mathbf{3}}$ & $\mathbf{1 0}^{\mathbf{4}}$ & $\mathbf{1 0}^{\mathbf{5}}$ \\
\hline Present study & 1.099 & 2.295 & 4.664 \\
Davis (1983) & 1.118 & 2.243 & 4.519 \\
Nithyadevi et al. (2009) & 1.123 & 2.304 & 4.899 \\
\hline
\end{tabular}

Table 2. Validation of the numerical results.

\begin{tabular}{ccccc}
\hline Ra & $\mathcal{E}$ & Present Results & Muthtamilselvan et al. (2009) & June (2001) \\
\hline \multirow{2}{*}{$10^{5}$} & 0.4 & 2.9482 & 2.9004 & 2.9435 \\
& 0.6 & 3.1310 & 3.0893 & 3.0877 \\
\hline
\end{tabular}




\section{Results and Discussion}

This section illustrates the influence of the Darcy number (Da), Hartmann number (Ha), Reynolds number $(\mathrm{Re})$, Rayleigh number $(\mathrm{Ra})$ and the Forchheimer parameter $(\mathrm{F} \varepsilon)$, on the irreversibility, heat transfer and flow structure of a Darcy-Brinkman-Forchheimer fluid flow $(\mathrm{Pr}=7)$ in a lid-driven porous cavity. The dimensionless numbers listed above were ranging from $10^{-3}$ to 1 , from 0 to 100 , from 10 to 50 , from $10^{4}$ to $10^{5}$ and from 0.25 to 0.87 respectively. The porosity $(\varepsilon)$ of the media is fixed at 0.5. The modified Brinkman number is fixed at $5 \times 10^{-7}$.

The streamlines, isotherms and isentropic lines at steady state are shown in Figure 2 for different Hartmann numbers ( $\mathrm{Ha}=0,50$ and 100$)$. As can be seen in Figure $2 \mathrm{a}$, the flow is characterized by the predominance of one cell at low $(\mathrm{Ha})$. As Ha increases $(\mathrm{Ha}>50)$, the flow structure changes and takes the shape of two cells. This change in the flow structure is accompanied by a decrease in the flow velocity, which is the result of the decelerator effect of the Lorentz force via the magnetic field. As seen in Figure 2b, isotherms are strongly deformed near the top wall at zero Hartmann number. This is due to the inertial force resulting from the driven wall. As (Ha) increases, the deformation of the isotherms gradually decreases and becomes practically absent in the right side of the cavity. As seen in Figure 2c, the upper half of the cavity is not engendered in entropy generation. Isentropic lines are tightened close to the hot wall, where the thermal gradients are important.

Figures 3 and 4 show the effect of both (Da) and (Ha), on the average Nusselt number $(\mathrm{Nu})$ and the total entropy generation $(S)$ respectively. Results show that $(\mathrm{Nu})$ and $(S)$ decrease with the decrease of (Da). In fact, when decreasing (Da) from unity, the permeability of the porous medium decreases and therefore the convection reduces. This reduction leads to a decrease in the thermal and velocity gradients, which induces a decrease in both $(\mathrm{Nu})$ and $(\mathrm{S})$. At fixed $(\mathrm{Da})$, Figures 3 and 4 show a decrease of $(\mathrm{Nu})$ and $(S)$ when $(\mathrm{Ha})$ increases. This is due to the retarding effect of the Lorentz force on the flow. The influence of the Reynolds number on the Nusselt number and the entropy generation is depicted in Figures 5 and 6 . At a fixed Hartman number, Figure 5 shows that $(\mathrm{Nu})$ increases with the increase of $(\mathrm{Re})$. This could be the result of an increase in the form drag related to the Forchheimer effect in the porous medium. Note that the increase of the Nusselt number and the entropy generation is less important as (Ha) is important. In this case, for important $(\mathrm{Ha})$, the Lorentz force becomes significant enough to be able to reduce the form drag effect. Similar observations to those of Figure 4 can be conducted for Figure 6, related to the variation of the entropy generation versus the Reynolds number. It is important to notice that the irreversibility in the lid-driven cavity is principally caused by the heat transfer, since the chosen value of the modified Brinkman number is small.
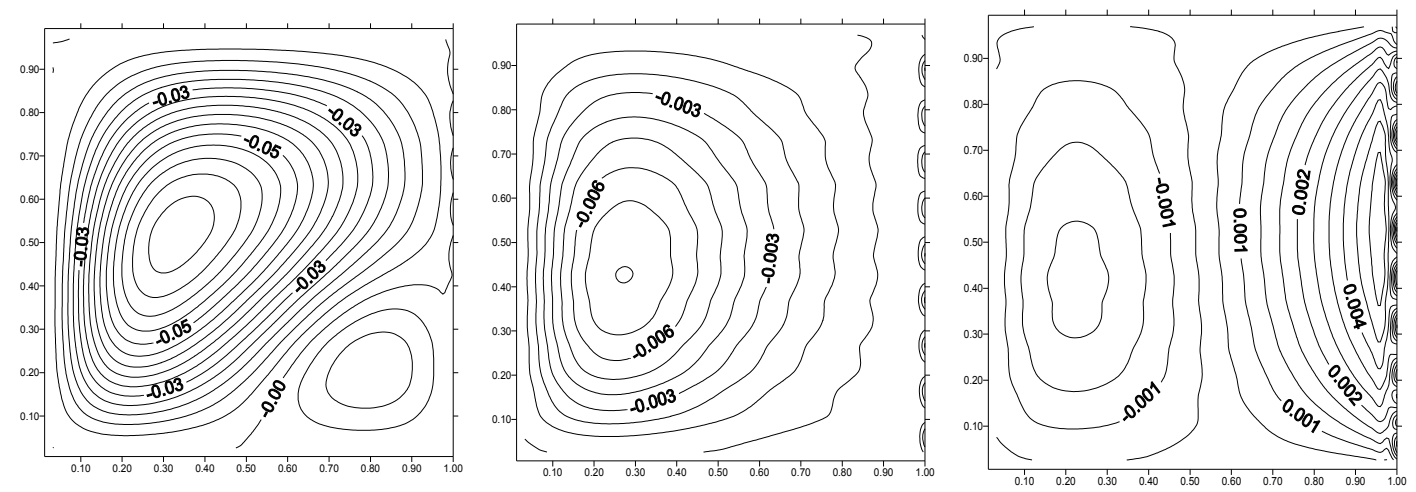

(a)

Figure 2. Cont. 

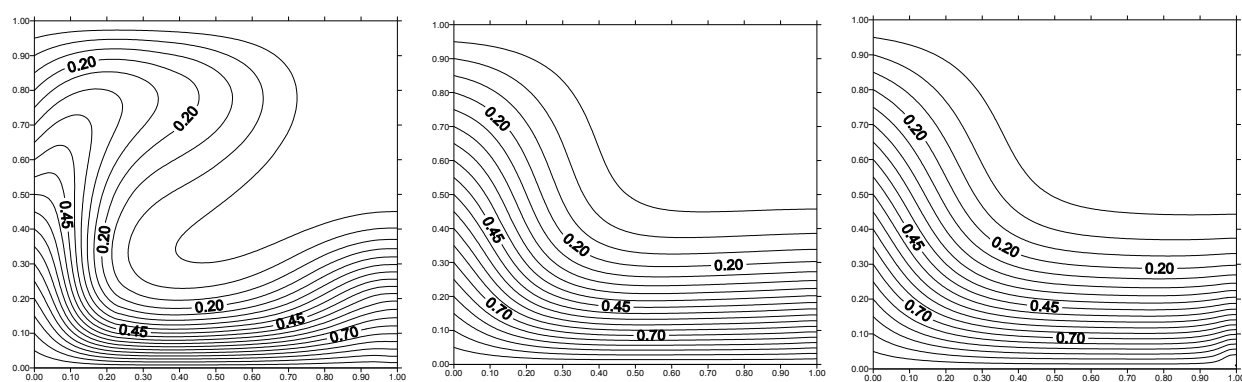

(b)
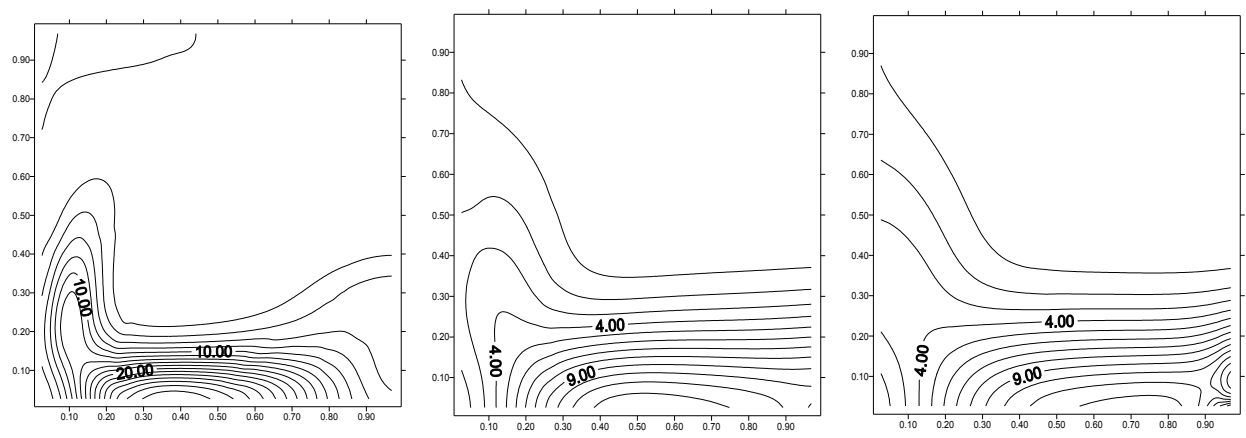

(c)

Figure 2. (a) Streamlines; (b) Isotherms; (c) Isentropic lines $\left(\operatorname{Re}=10, \mathrm{Ra}=10^{5}, \mathrm{Da}=10^{-2}\right)$.

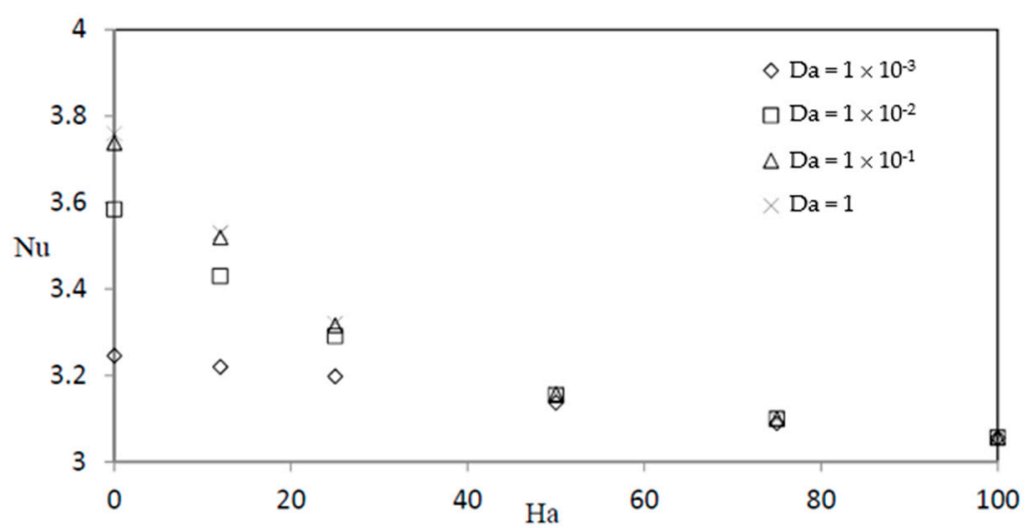

Figure 3. Average Nusselt number versus Hartmann number for different Darcy numbers $\left(\operatorname{Ra}=10^{5}\right.$, $\operatorname{Re}=10)$.

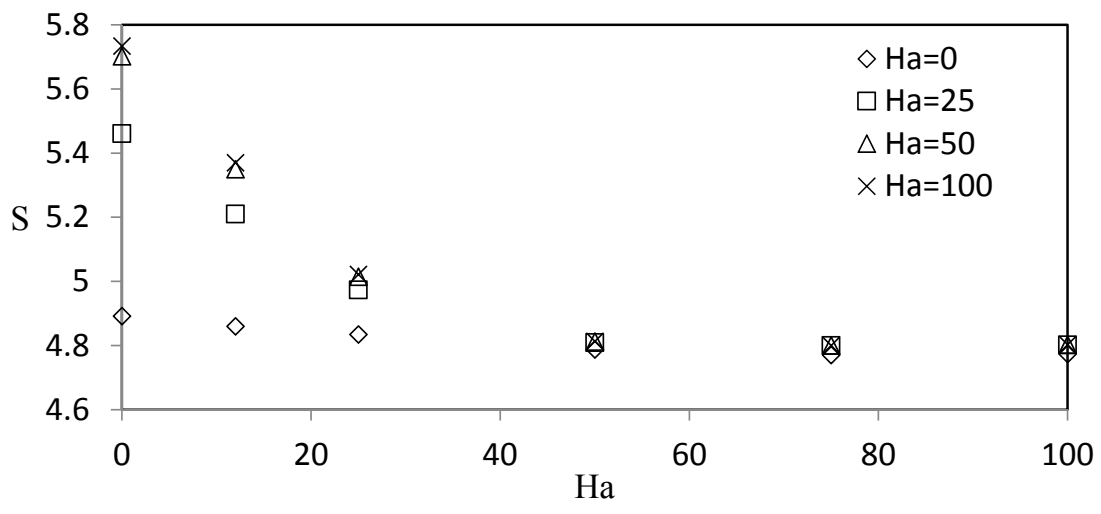

Figure 4. Entropy generation rate versus Hartmann number for different Darcy numbers $\left(\operatorname{Ra}=10^{5}\right.$, $\operatorname{Re}=10$ ). 


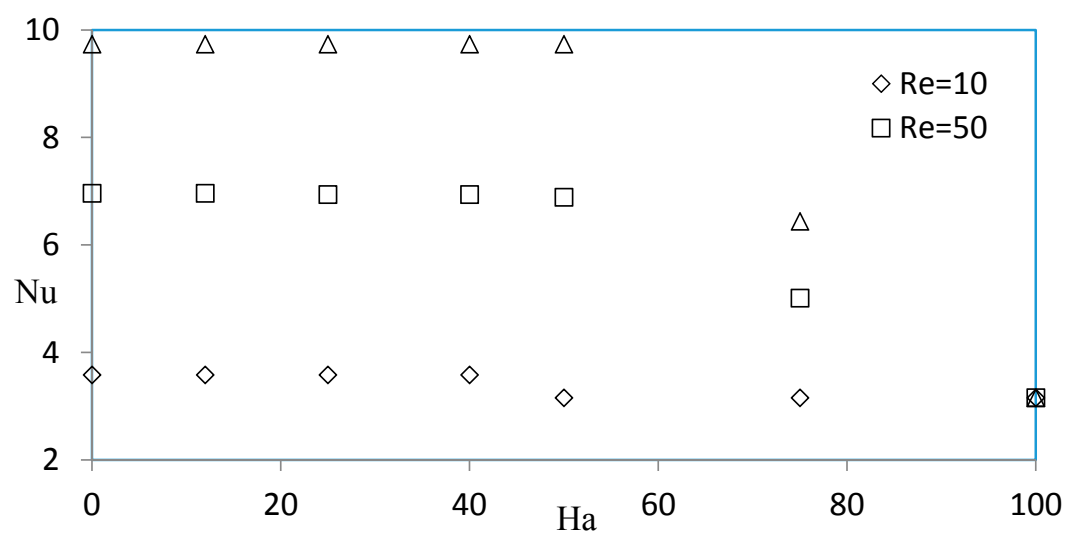

Figure 5. Average Nusselt number versus Hartmann number for different Reynolds numbers $\left(\mathrm{Ra}=10^{5}, \mathrm{Da}=10^{-2}\right)$.

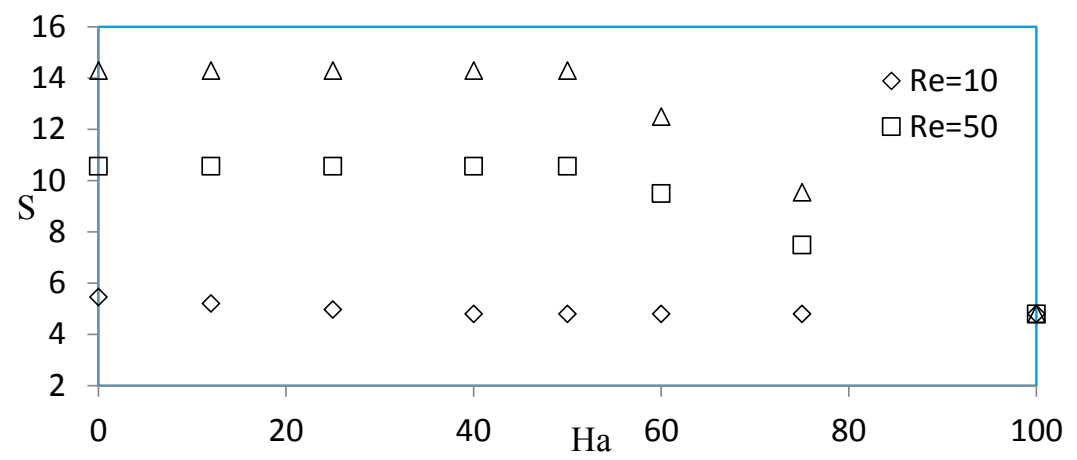

Figure 6. Entropy generation rate versus Hartmann number for different Reynolds numbers $\left(\operatorname{Ra}=10^{5}\right.$, $\left.\mathrm{Da}=10^{-2}\right)$.

Figure 7 illustrates the evolution of the total entropy generation with the Hartman number for $\mathrm{Ra}=10^{4}$ and $10^{5}$. As shown in Figure 7, at a fixed Hartmann number, the entropy generation generally increases as the Rayleigh number increases. This is due to the improvement of the convection in the porous cavity which will, in turn, increase the thermal and the velocity gradients and consequently the entropy generation.

Figure 8 illustrates the Forchheimer parameter effect on the entropy generation when (Ha) progresses from 0 to 100 . Since the modified Brickman number value is very small, there is virtually no intrinsic effect of (Ha) via the magnetic and the Darcy viscous irreversibility terms on the total entropy generation (Equation (10)). Consequently, the effect of (Ha) on the total entropy generation is principally extrinsic through the momentum balance equation. Figure 8 shows that for (Ha) ranging from 0 to 25, the total entropy generation takes a constant value depending on the Forchheimer parameter. When (Ha) increases from 25 to 50 , the total entropy generation decreases and converges towards the same constant value close to 4.8 for all selected Forchheimer parameters. For (Ha) exceeding 50, no significant effect of the Forchheimer parameter is observed and the entropy generation remains almost constant. These observations show the existence of three flow regimes. The first is the Forchheimer regime which persists until a critical Hartmann number $\mathrm{Ha}_{\mathrm{c}}=25$, for which the form drag linked to the non-linear velocity term is important. The second is similar to the Darcy regime for (Ha) greater than 50. In this case, the fluid undergoes a deceleration due to the dominant effect of the Lorentz force and consequently the form drag becomes insignificant. The third regime, for (Ha) between 25 and 50, is transitional from the first where the form drag is dominant to the second where the Lorentz force is dominant. 


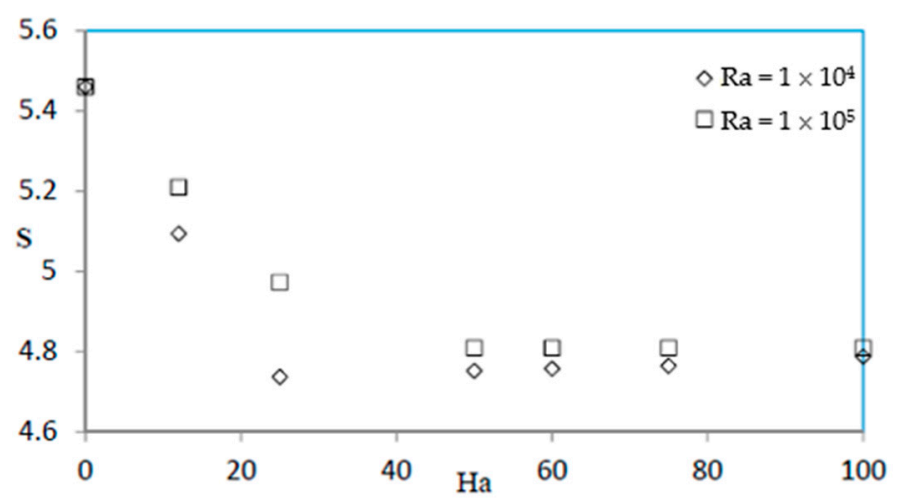

Figure 7. Entropy generation rate versus Hartmann number for $\mathrm{Ra}=10^{4}$ and $\mathrm{Ra}=10^{5}(\mathrm{Re}=10$, $\left.\mathrm{Da}=10^{-2}\right)$.

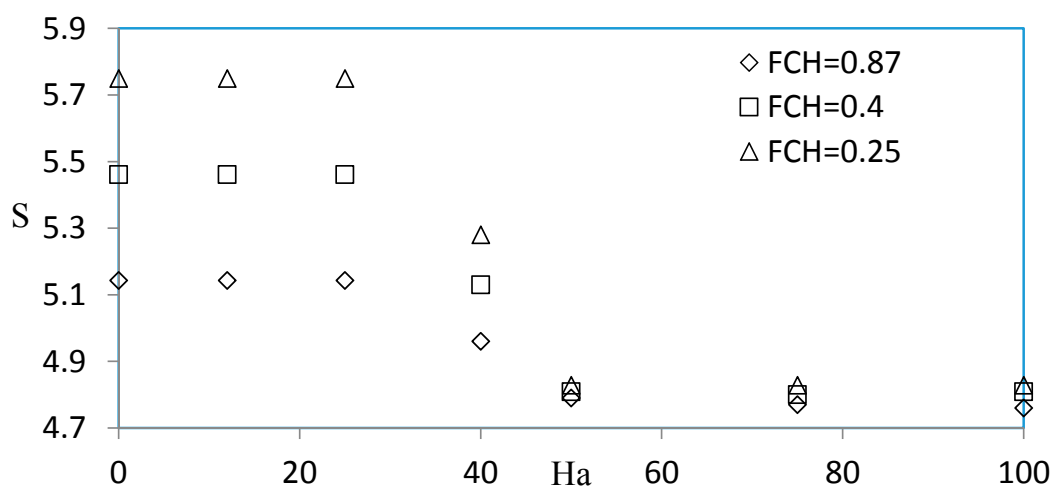

Figure 8. Entropy generation rate versus Hartmann number for three Forchheimer parameter values of $0.25,0.4$ and $0.87\left(\mathrm{Ra}=10^{5}, \mathrm{Da}=10^{-2}\right)$.

\section{Conclusions}

Entropy generation and heat transfer in MHD mixed convection in a porous lid-driven cavity submitted to a vertical temperature gradient were studied. The Navier-Stokes and energy equations written in the Darcy-Brinkman-Forchheimer formulation were solved by using CVFEM numerical code. The effect of the Darcy, Rayleigh, Reynolds, Hartmann numbers and the Forchheimer parameter on heat transfer and entropy generation were investigated. Main conclusions are:

(1) The entropy generation rate decreases with the decrease of Darcy number and the increase of Hartmann number.

(2) The flow structure strongly depends on the Hartmann number.

(3) At a fixed Hartmann number, the entropy generation increases with the increase of the Reynolds number.

(4) At a fixed and relatively high Reynolds number, the entropy generation remains constant for a Hartmann number smaller than 50 .

(5) The Forchheimer effect is significant for a Hartmann number between 0 and 25 and negligible for a Hartmann number greater than 50.

Acknowledgments: I have to express our appreciation to Mourad Magherbi and Ammar Ben Brahim for their scientific support and their valuable comments.

Author Contributions: Rahma Bouabda and Mounir Bouabid performed the numerical code and validated the numerical results. Rahma Bouabda and Mourad Magherbi analyzed and discussed the results. Rahma Bouabda and Ammar Ben Brahim wrote the paper.

Conflicts of Interest: The authors declare no conflict of interest. 


\section{Nomenclature}

$\mathrm{B}_{0} \quad$ uniform magnetic field

$\mathrm{Br} \quad$ Brinkman number $\mathrm{Br}=\mu \mathrm{U}_{0}^{2} /\left(\lambda_{\mathrm{e}} \Delta \mathrm{T}\right)$

$\mathrm{Br}^{*} \quad$ modified Brinkman number $\mathrm{Br}^{*}=\mathrm{Br} / \Omega$

$\mathrm{C}_{\mathrm{p}} \quad$ specific heat $\left(\mathrm{J} \cdot \mathrm{Kg} \cdot \mathrm{K}^{-1}\right)$

$\mathrm{Da} \quad$ Darcy numberk $/ \mathrm{H}^{2}$

g gravitational acceleration $\left(\mathrm{m} \cdot \mathrm{s}^{-2}\right)$

$J_{k} \quad$ diffusion flux $(k=U, V, T, \mathrm{C})$

$\mathrm{K}$ permeability of the medium $\left(\mathrm{m}^{2}\right)$

$\mathrm{Nu} \quad$ Nusselt number

$P \quad$ dimensionless pressure

Pr Prandtl number

$\mathrm{Ra} \quad$ Rayleigh number

Re Reynolds number

$\mathrm{Ri} \quad$ Richardson number

$U$ dimensionless velocity vector

$U, V \quad$ dimensionless velocity components

$T \quad$ Dimensionless temperature $T=\left(\theta-\theta_{\mathrm{c}}\right) /\left(\theta_{\mathrm{h}}-\theta_{\mathrm{c}}\right)$

$X, Y \quad$ dimensionless Cartesian coordinates

Greek letters

$\tau \quad$ dimensionless time

$\alpha \quad$ thermal diffusivity $\lambda_{\mathrm{e}} /\left(\rho \mathrm{C}_{\mathrm{p}}\right)$,

$\beta_{\theta} \quad$ thermal expansion coefficient

$\rho \quad$ mass density

$\Theta \quad$ temperature

$\mu \quad$ dynamic viscosity

$\checkmark \quad$ kinematic viscosity $\left(\mathrm{m}^{2} \cdot \mathrm{s}^{-1}\right)$

$\varepsilon \quad$ porosity of the media

$\nabla \quad$ Nabla vector

\section{References}

1. Nield, D.A.; Bejan, A. Convection in Porous Media, 3rd ed.; Springer: New York, NY, USA, 2006.

2. Ingham, D.B.; Pop, I. Transport Phenomena in Porous Media; Elsevier: Oxford, UK, 2005.

3. Vafai, K. Handbook of Porous Media, 2nd ed.; Taylor \& Francis: Boca Raton, FL, USA, 2005.

4. Bejan, A.; Dincer, I.; Lorente, S.; Miguel, A.F.; Reis, A.H. Porous and Complex Flows Structures in Modern Technologies; Springer: New York, NY, USA, 2004.

5. Chamkha, A.J. Hydromagnetic combined convection flow in a vertical lid-driven cavity with internal heat generation or absorption. Numer. Heat Transf. A Appl. 2002, 41, 529-546. [CrossRef]

6. Khanafer, K.; Vafai, K. Double-diffusive mixed convection in a lid-driven enclosure filled with a fluid saturated porous medium. Numer. Heat Transf. A Appl. 2002, 42, 465-486. [CrossRef]

7. Cheng, T.S. Characteristics of mixed convection heat transfer in a lid-driven square cavity with various Richardson and Prandtl numbers. Int. J. Ther. Sci. 2011, 50, 197-205. [CrossRef]

8. Muthtamilselvan, M.; Kandaswamy, P.; Lee, J. Hydromagnetic mixed convection in a lid-driven cavity filled with a fluid-saturated porous medium. Int. J. Appl. Math. Mech. 2009, 5, 28-44.

9. Rahman, M.M.; Billah, M.M.; Mamun, M.A.H.; Saidur, R.; Hasanuzzaman, M. Reynolds and Prandtl numbers effects on MHD mixed convection in a lid-driven cavity along with joule heating and a centered heat conducting circular block. Int. J. Mech. Mater. Eng. 2010, 5, 163-170.

10. Mansour, M.A.; Mohamed, R.A.; Abd-Elaziz, M.M.; Ahmed, S.E. Numerical simulation of mixed convectionflows in a square lid-driven cavity partially heated from below using nanofluid. Int. Commun. Heat Mass Transf. 2010, 37, 1504-1512. [CrossRef]

11. Saha, L.K.; Somadder, M.C.; Roy, N.C. Hydro-magnetic mixed convection flow in a lid-driven cavity with wavy bottom surface. Am. J. Appl. Math. 2015, 3, 8-19.

12. Mamourian, M.; Shirvan, K.M.; Ellahi, R.; Rahimi, A.B. Optimization of mixed convection heat transfer with entropy generation in a wavy surface square lid-driven cavity by means of Taguchi approach. Int. J. Heat Mass Transf. 2016, 102, 544-554. [CrossRef] 
13. Akbar, N.S.; Raza, M.; Ellahi, R. Endoscopic effects with entropy generation analysis in peristalsis for the thermal conductivity of $\mathrm{H}_{2} \mathrm{O}+\mathrm{Cu}$ nanofluid. J. Appl. Fluid Mech. 2016, 9, 1721-1730.

14. Ellahi, R.; Hassan, M.; Zeeshan, A. Shape effects of nanosize particles in $\mathrm{Cu}-\mathrm{H}_{2} \mathrm{O}$ nanofluid on entropy generation. Int. J. Heat Mass Transf. 2015, 81, 449-456. [CrossRef]

15. Akbar, N.S.; Raza, M.; Ellahi, R. Peristaltic flow with thermal conductivity of $\mathrm{H}_{2} \mathrm{O}+\mathrm{Cu}$ nanofluid and entropy generation. Results Phys. 2015, 5, 115-124. [CrossRef]

16. Van Doormal, J.P.; Raithby, G.D. Enhancements of the SIMPLE method for predicting incompressible fluid flow. Numer. Heat Transf. 1984, 7, 147-163. [CrossRef]

17. Patankar, S.V. Numerical heat transfer and fluid flow. In Computational Methods in Mechanics and Thermal Sciences; Hemisphere/Mac Graw-Hill: New York, NY, USA, 1980.

18. Abbassi, H.; Turki, S.; Nasrallah, S.B. Mixed convection in a plane channel with a built-in triangular prison. HTPA 2001, 39, 307-320.

19. Saabas, H.J.; Baliga, B.R. Co-located equal-order control volume finite element method for multidimensional incompressible fluid flow-Part II: Verification. Numer. Heat Transf. B Fundam. 1994, 26, 381-407. [CrossRef]

20. Pantakar, S.V.; Spalding, D.B. A calculation procedure for heat, mass and momentum transfer in three dimensional parabolic flows. Int. J. Heat Mass Transf. 1972, 15, 1787-1806.

21. Jue, T.C. Analysis of Bénard convection in rectangular cavities filled with a porous medium. Acta Mech. 2001, 146, 21-29. [CrossRef]

(C) 2016 by the authors; licensee MDPI, Basel, Switzerland. This article is an open access article distributed under the terms and conditions of the Creative Commons Attribution (CC-BY) license (http://creativecommons.org/licenses/by/4.0/). 